\title{
Indefinites between Predication and Reference
}

\author{
Henriëtte de Swart \\ Utrecht University
}

\begin{abstract}
The literature on indefinites uses different criteria to characterize different subclasses of NPs as 'indefinites'. This paper develops an analysis of weak and referential NPs in the flexible type-theoretical framework developed by Partee (1987). All weak NPs have a type $\langle e, t\rangle$ denotation, and can be shifted back onto their original generalized quantifier denotation by applying the appropriate inverse operation of BE. Referential NPs are defined as the subset of weak NPs which allow mappings onto a type $e$ denotation. This mapping is dependent on the partition of the domain of individuals salient in the context of the discourse.
\end{abstract}

\section{Three characterizations of indefinite NPs}

In the literature on noun phrases (NPs), indefinites are frequently treated as a separate class, and have been subject to special attention. However, in different contexts, different definitions and criteria are used, and the resulting classifications are not always the same. Thus, it turns out that the term 'indefinite' is used for a morphologically, syntactically and semantically heterogeneous group. In the literature, we find three main characterizations of indefinite NPs, based on the weak/strong distinction, dynamic binding, and predication.

I. Weak/strong distinction

Indefinites are weak in the sense of the weak/strong distinction (Milsark 1977, Barwise and Cooper 1981). Weak quantifiers can be felicitously used in existential contexts such as there-sentences, whereas strong quantifiers cannot:

(1) Existential contexts

a. There is a cat/ no cat/ *the cat/ *every cat/ *neither cat in the garden

b. There are cats/ two cats/ many cats/ no cats/ at most two cats/ ${ }^{*}$ the cats/ *all cats/ *most cats in the garden 
This criterion leads us to characterize NPs like a cat, books, many pencils, three linguists, at least four children, exactly two students, less than four babies, few tables, no articles, etc. as weak, and NPs like the cat, all students, every book, most pencils, neither linguist, etc. as strong. Weakness has been analyzed in terms of cardinality, symmetry, intersectivity or existentiality (cf. Milsark 1977, Barwise and Cooper 1981, Keenan 1987, Blutner 1993, Zucchi 1996, McNally 1998 to mention just a few of the proposals that have been made in the literature). The various notions have in common that the cardinality of just one set (typically, the intersection of the restrictor set and the scope of the determiner) is sufficient to determine the truth conditions of the sentence. Various other criteria pick out the same class of NPs. Milsark (1977) observed that indefinites get either a weak or a strong reading in the subject position of a stage-level predicate. Indefinite subjects of individual-level predicates always get a strong reading, compare:

(2) Stage-level predicates

a. A student called.

b. Dogs are playing in the front yard.

c. Two children are waiting for the bus.

d. No train arrived.

e. Few children are playing in the street.

(3) Individual-level predicates

a. A cousin of mine is in the navy. [specific]

b. Students are intelligent. [generic]

c. Two students like semantics. [partitive]

d. No cat likes milk [generic]

e. Few students like semantics [partitive/proportional]

Furthermore, weak NPs are felicitous in the object position of verbs like travel and have (4a), (5a). Strong NPs are excluded from this position (4b), (5b) (compare de Jong 1987, de Hoop 1992). De Hoop (1992) analyzes this as a form of semantic incorporation:

(4) a. Ik heb gisteren enkele/ vele/ meer dan twintig/ minder dan twintig. . kilometer afgelegd [Dutch] I have yesterday some/many/more than twenty/less than twenty... kilometers travelled 
b. *Ik heb gisteren alle/niet alle/de meeste/... kilometers afgelegd I have yesterday all/not all/most/... kilometers travelled

(5) a. The house has windows/at least two windows/many windows/no windows/less than five windows ...

b. ${ }^{*}$ The house has all windows/most windows/neither window...

Finally, Zimmermann (1993) observes that the de re/de dicto distinction can be established with weak NPs, but strong NPs always get a de re interpretation, compare:

(6) intensional verbs

a. Eve seeks a new book on semantics/ two students interested in semantics/ no unicorns [de re/ de dicto]

b. Eve seeks every new book on semantics/ most students interested in semantics/neither unicorn

[de re only]

According to Zimmermann, seek does not take generalized quantifiers in object position, but expressions of type $\langle e, t\rangle$. Weak NPs, which can be of this type, can stay in situ and get a de dicto reading. Strong NPs, which only have a generalized quantifier denotation, have to scope out and get a de re reading.

\section{Referentiality}

Indefinites are said to introduce discourse referents (Kamp 1981, Heim 1982 and many others). Expressions which introduce discourse referents allow dynamic binding. That is, they license discourse anaphora (binding across sentences), and so-called donkey anaphora (binding across a quantificational boundary). Compare:

(7) discourse anaphora

a. A student ${ }_{i}$ called. She ${ }_{i}$ had a question about the exam.

b. Every student $t_{i}$ called. \# She ${ }_{i}$ had a question about the exam.

c. No student ${ }_{i}$ called. \# She ${ }_{i}$ had a question about the exam.

d. The chair ${ }_{i}$ of the department called. She $_{i}$ wanted to make an appointment.

The infelicity of (7c) shows that no student should be classified with NPs like every student, rather than a student as far as its licensing properties are concerned. This means that the set of indefinite NPs which license discourse anaphora (class II) is a proper subset of the set of indefinites which have 
weak readings (class I). Groenendijk and Stokhof (1989) argue that monotone deacreasing (mon $\downarrow$ ) NPs do not license discourse anaphora, because that would create a 'downdate' rather than an 'update' of information. Thus no N cannot be characterized as an indefinite NP in the Kamp/Heim sense of the term. But the situation is more complex. Note first that we cannot say that the set of NPs which license discourse referents is a proper subset of the set of NPs which are felicitous in existential contexts. It is often assumed that strong NPs, including definite NPs are not very good in existential contexts (1a), although there are in fact systematic exceptions to this rule, as shown by McNally (1998). Furthermore, definite NPs are commonly accepted as licensors of discourse anaphora (7d). Thus, the property which characterizes group II indefinites is not inherently related to the notion of weakness, which characterizes group I indefinites.

If we extend the discussion to plural NPs, we observe that not all weak NPs which are not downward entailing behave like indefinite NPs in the Kamp/Heim sense of the term. Although the non-monotone and monotone increasing weak NPs in (8b) and (c) license discourse anaphora, the binding relation is not the same as in $(8 \mathrm{a})$ :

(8) a. Two students came in. They had a question about the exam.

b. At least two students came in. They had a question about the exam.

c. Exactly two students came in. They had a question about the exam.

d. All students came to the meeting. They requested a new class schedule.

As pointed out by Kamp and Reyle (1993), the bare numeral NP in (8a) introduces a plural discourse referent which sets up an anchor for further reference. They point out that in $(8 \mathrm{~b})$ and (c), it is not the cardinal NP itself which creates the antecedent for the plural pronoun they, but the sentence as a whole. In order to interpret they, we build a group referent for the entire set of students who came in. As emphasized by Szabolcsi (1997a: 25), we cannot continue $(8 \mathrm{~b})$ with the sentence 'Perhaps there were others who did the same'. However, we can do this in (8a). This shows that they just refers to the two students the speaker was talking about in the first sentence, and there might in fact be other students around in the domain of discourse. Thus, bare numeral NPs allow non-maximal anaphora, just like singular indefinites. The fact that this continuation is infelicitous in $(8 b)$ and (c) illustrates that modified numerals only allow maximal anaphora. The sum formation which Kamp and Reyle (1993) define for the cases in (8b) and (c) extends to other plural quantifiers, as shown by (8d). In the plural domain, we thus need to distinguish two different mechanisms of licensing discourse anaphora, only one of which runs parallel to the singular case. 
This criterion leads to the characterization of NPs like a student, three linguists, some books, many tables as indefinite. NPs such as no student, at least three students, exactly four books, less than three linguists no longer count as indefinites. The semantic property which underlies this behavior is referentiality, or the fact that the expression in some sense characterizes an individual or a group as a distinguished entity. Related criteria which pick out roughly the same class are: island escaping behavior (scope outside of a quantificational domain) (Farkas 1981, 1997, Abusch 1994, Ruys 1993, Reinhart 1997, Winter 1998), wide scope of object NPs with respect to subject NPs (Beghelli and Stowell 1997, Szabolcsi 1997b), permissible interveners in weak island constructions (Honcoop 1998).

\section{Predication}

Both the literature on the weak/strong distinction and the work on reference and anaphora have argued that indefinites are just predicates, so semantically speaking they are not really full NPs. The criterion is mainly occurrence with predicative be. Interestingly, if we take a closer look at this criterion, we observe that this construction really picks out a third class of indefinites. The examples in (9) show that predicative be is compatible with a subset of class I indefinites only. Furthermore, not all type II indefinites occur in this construction, whereas some indefinites which are not in class II do:

(9) predicative be

a. Sara is a well-known linguist.

b. John is no genius.

c. The apostles are twelve.

d. ??Sara and John are two first-year students.

e. Sara and John are two of our most promising students.

f. *Sara and John are at least/at most two students.

g. Sara is the chair of the department.

Predicative be is compatible with class II indefinites like a linguist (9a), but also with the class I indefinite no genius (9b). Examples with no $\mathrm{N}$ in the complement of predicative be $(9 \mathrm{~b})$ are not very common in English, but they are widespread in Germanic languages like Dutch and German (compare de Swart, to appear). Bare numerals are good class II indefinites, but are somewhat restricted in the context of predicative $b e$. The bare predicative numeral in (9c) (from Blutner 1993) is fine, but the full numeral NP in (9d) is strange. Embedding in a partitive construction considerably improves the situation (9e) 
(from Donka Farkas, p.c.). Other class I indefinites such as modified numerals are clearly excluded from constructions involving predicative be (9f). Definite NPs are compatible with predicative be $(9 \mathrm{~g})$, so this criterion is also not sensitive to indefiniteness as such. The distribution in (9) makes it clear that predicative be is not sensitive to the weak/strong distinction. Because of the felicity of no $\mathrm{N}$ and the complexities introduced by bare numerals, it cannot be crucially dependent on referentiality either. Intuitively, the crucial underlying property is qualitative rather than quantitative predication.

The three classes of NPs discussed here show that the definition of what characterizes an indefinite NP is dependent on the semantic phenomenon at issue, and the criteria used as a basis for the classification. We accept this as entirely legitimate, but we are interested in finding out how the different notions of indefiniteness are related, and how we can develop an analysis which gives the different subclasses a coherent semantic foundation. In the following, we will refer to the three classes as Class I, II and III indefinites, and label their characterizing properties as weakness, referentiality and qualitative predication:

- Class I indefinites: weakness

- Class II indefinites: referentiality

- Class III indefinites: qualitative predication

We will not be concerned with the analysis of Class III indefinites in this paper, but refer the interested reader to de Swart (1999) for a proposal. The aim of this paper is to give semantic content to the properties of weakness and referentiality in such a way that we capture the systematic relations between the different classes of indefinites. Interestingly, many of the authors quoted above use a flexible type-theory as their semantic framework (compare De Hoop 1992, Blutner 1993, Zimmermann 1993, Chierchia 1998 and others), following ideas developed by Partee (1987). Partee argues that some NPs have a family of denotations in different types, and that natural language allows various shifts between type $e$, type $\langle e, t\rangle$ and type $\langle\langle e, t\rangle, t\rangle$ expressions. The type-shifting perspective is the starting point of our investigation.

Section 2 uses Partee's type-shifter BE to define class I NPs as those NPs which have a well-formed denotation in the domain of expressions of type $\langle e, t\rangle$, which is neither the empty set, nor a singleton set. Thus, weak NPs are predicative. Section 3 defines three inversion operators which allow a mapping of type $\langle e, t\rangle$ expressions back onto the original generalized quantifier type $\langle\langle e, t\rangle, t\rangle$. This allows us to maintain the claim that weak NPs are predicative, and at the same time preserve function application as the only mode of composition at the sentence level. Section 4 argues that referentiality needs to be defined in terms of a mapping onto a well-formed expression of type $e$. The mapping is dependent on a partioning of the domain of individuals in a lattice-theoretical 
ontology of plurals. NPs which have a well-formed denotation of type $e$ are externally dynamic, NPs which do not are externally static. Section 5 shows that we find more dynamics than we thought at first sight. We argue that the partitioning of the domain of individuals, and thereby the externally dynamic behavior of indefinites is dependent on the context at hand. In sum, the claims made in this paper are the following:

- Class I indefinites are those NPs which, after application of BE have a well-formed denotation in the domain of expressions of type $\langle e, t\rangle$, which is neither the empty set, nor a singleton set.

- The type $\langle e, t\rangle$ denotation of an NP can be shifted back onto its original type $\langle\langle e, t\rangle, t\rangle$ denotation by applying existential insertion $(\exists \mathrm{I})$, universal insertion $(\forall \mathrm{I})$ or exact existential insertion $(\exists ! \mathrm{I})$, depending on the monotonicity properties of the underlying generalized quantifier (monotone increasing, monotone decreasing, or non-monotone respectively).

- Class II indefinites are the subset of Class I indefinites which can be mapped onto a well-formed expression of type $e$. This mapping is dependent on the partition of individuals salient in the context.

\section{Deriving a property denotation}

In section 1, we observed that existential contexts, subject positions of stagelevel predicates and object positions of semantically incorporating verbs are sensitive to the weak/strong distinction. These contexts provide evidence that weak NPs are somehow different from strong NPs. In the literature, strong NPs are characterized as real quantifiers, and weak NPs are not. This gives rise to the first important generalization: indefinite NPs are non-quantificational, or at least, not necessarily quantificational NPs. In a type-theoretical perspective, real quantifiers are usually interpreted as generalized quantifiers, that is, expressions of type $\langle\langle e, t\rangle, t\rangle$. In the literature, we find essentially two views on how to obtain a property denotation for indefinite NPs. One option is to postulate that $\langle e, t\rangle$ is the basic type of an indefinite. This can be regarded as the view adopted by Discourse Representation theory (Kamp 1981, Heim 1982, Kamp and Reyle 1993), but also the choice function approach (in particular Reinhart 1997, Winter 1997, Kratzer 1998). One problem for this approach is the diversity of the notion of indefiniteness presented in section 1, which raises the question: which indefinites are basically predicates, and why? Others have proposed to derive the predicative interpretation via the type-shifting operation BE in Partee's (1987) flexible type theory. Examples are Zimmermann (1993), Blutner (1993), and partly McNally (1998). An important advantage of this approach is that the weak reading of the indefinite NP can be derived in a straightforward way from the standard generalized quantifier denotation 
of the NP. The view I will defend here is that application of BE helps us to define the set of class I indefinites.

In Partee's (1987) flexible type-theory, all NPs have an interpretation in the domain of expressions of type $\langle\langle s,\langle e, t\rangle\rangle, t\rangle$. This is the case for NPs which have denotations of type $e$ or $\langle e, t\rangle$ as well. Such NPs get a family of types as their interpretation. Quantificational NPs are defined as those NPs which only have a denotation of type $\langle\langle s,\langle e, t\rangle\rangle, t\rangle$. Non-quantificational NPs have denotations in the domain of expressions of type $e$ ('referential') or $\langle e, t\rangle$ ('predicative') as well as of type $\langle\langle s,\langle e, t\rangle\rangle, t\rangle$ ('quantificational'). Type-shifting principles mediate between the different denotations of an NP. In such a perspective, it is important to determine which NPs have well-formed denotations of type $e$ and $\langle e, t\rangle$, and which type-shifting principles natural languages use to map denotations of a certain type onto denotations of some other type. In order to avoid overgeneration, only 'natural' type-shifting operations are allowed. The general picture is sketched in figure 1:

\section{figure 1}

A family of interpretations for NPs

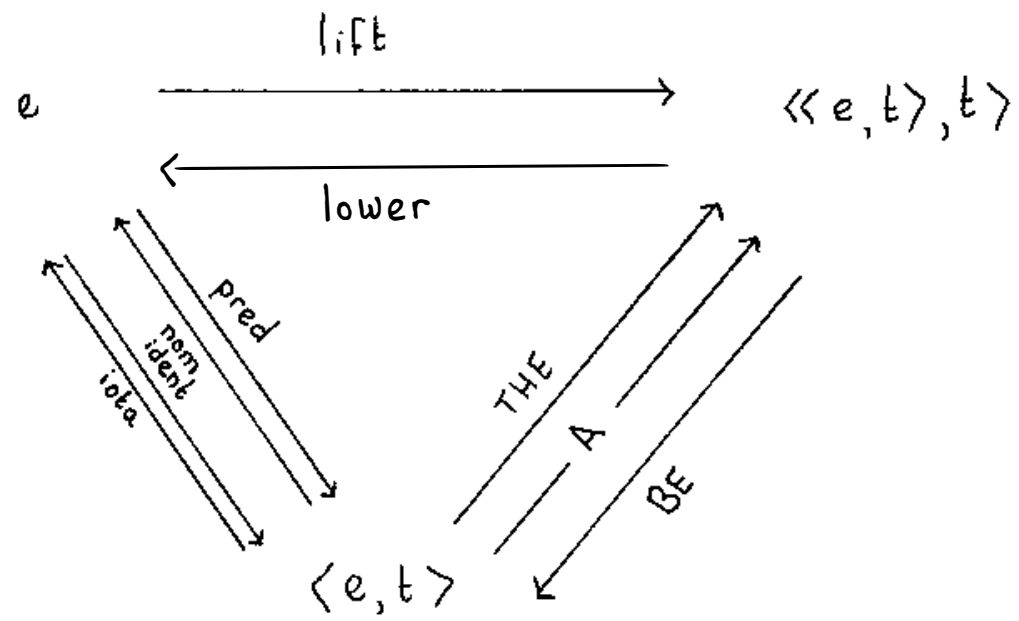

One of the natural type-shifting operations Partee discusses is the mapping $\mathrm{BE}$, relating generalized quantifier denotations and predicative interpretations:

(10) $\mathrm{BE}: \lambda \mathcal{P} \lambda x\left({ }^{\vee} \mathcal{P}(\wedge \lambda y[y=x])\right)$

where $\mathcal{P}$ corresponds with a variable of type $\langle s,\langle\langle s,\langle e, t\rangle\rangle, t\rangle$,

$\mathrm{BE}$ finds all the singleton sets in the generalized quantifier denotation and collects them into a set. BE is a total function, but yields the empty set when the generalized quantifier does not contain singleton sets, as in the case of most students or every student (in a domain of more than one student). Indefinite NPs of the form $a N$ have well-formed predicative interpretations, for instance: 
(11) a student $\sim B E \mathrm{BE}$ (a student)

$\lambda Q \exists z$ (Student $\left.(z) \wedge{ }^{\vee} Q(z)\right) \sim{ }_{B E}$

$\lambda \mathcal{P} \lambda x\left({ }^{\vee} \mathcal{P}(\wedge \lambda y[y=x])\right)\left({ }^{\wedge} \lambda Q \exists z\left(\operatorname{Student}(z) \wedge{ }^{\vee} Q(z)\right)\right)$

$=\lambda x \lambda Q \exists z\left(\operatorname{Student}(z) \wedge{ }^{\vee} Q(z)\right)\left({ }^{\wedge} \lambda y[y=x]\right)$

$=\lambda x \exists z(\operatorname{Student}(z) \wedge[z=x])$

$=\lambda x(\operatorname{Student}(x))$

The arrow $\sim B E$ represents the type-shifting operation $\mathrm{BE}$, so that we have an expression of type $\langle\langle s,\langle e, t\rangle\rangle, t\rangle\rangle$ to the left of the arrow, and an expression of type $\langle e, t\rangle$ to the right. The derivation in (11) shows that we apply the interpretation of $\mathrm{BE}$ given in (10) to the generalized quantifier denotation of a student by means of function application. After successive lambda conversion and ${ }^{\vee} \wedge$-elimination, we end up with an expression of type $\langle e, t\rangle$ that characterizes the set of students in the model.

The following examples show that definite NPs and no $\mathrm{N}$ also have nonempty denotations in the domain of expressions of type $\langle e, t\rangle$. The derivations are standard, so I just give the original generalized quantifier denotation, and the predicative interpretation which results from application of $\mathrm{BE}$ after all the relevant lambda conversions and ${ }^{\vee} \wedge$-eliminations have been performed:

$$
\begin{aligned}
& \text { a. the chair } \sim \mathrm{BE}(\text { the chair }) \\
& \lambda Q \exists x\left(\text { Chair }(x) \wedge \forall u(\text { Chair }(u) \rightarrow[u=x]) \wedge{ }^{\vee} Q(x)\right) \sim B E \\
& \lambda x(\text { Chair }(x) \wedge \forall u(\text { Chair }(u) \rightarrow[u=x])) \\
& \text { b. no student } \sim_{B E} \mathrm{BE}(\text { no student }) \\
& \lambda Q \neg \exists z\left(\operatorname{Student}(z) \wedge{ }^{\vee} Q(z)\right) \sim B E \\
& \lambda \mathcal{P} \lambda x\left({ }^{\vee} \mathcal{P}\left({ }^{\wedge} \lambda y[y=x]\right)\right)\left({ }^{\wedge} \lambda Q \neg \exists z\left(\text { Student }(z) \wedge{ }^{\vee} Q(z)\right)\right) \\
& =\lambda x \neg(\text { Student }(x))
\end{aligned}
$$

In order to extend the analysis to plural NPs, we need to adopt a richer ontology. In Link's lattice-theory, plurals are non-atomic individuals, built out of atomic individuals by means of sum formation. If we treat plurals as complex individuals, we can apply a generalized version of the type-shifting operation $\mathrm{BE}$ to find the singleton sets of plural individuals in the denotation of the quantifier, and collect them into a set. For at least three students, at most three students, and exactly three students this leads to the well-formed predicative interpretations in (13):

(13) a. at least three students $\sim \mathrm{BE}$ (at least three students)

$\lambda Q \exists \gamma\left(\operatorname{Student}(\gamma) \wedge \operatorname{CARD}(\gamma) \geq 3 \wedge{ }^{\vee} Q(\gamma)\right) \sim_{B E}$

$\lambda \gamma(\operatorname{Student}(\gamma) \wedge \operatorname{CARD}(\gamma) \geq 3)$

b. at most three students $\leadsto \mathrm{BE}$ (at most three students)

$\lambda Q \neg \exists \gamma\left(\operatorname{Student}(\gamma) \wedge \operatorname{CARD}(\gamma)>3 \wedge{ }^{\vee} Q(\gamma)\right) \sim{ }_{B E}$

$\lambda \gamma \neg(\operatorname{Student}(\gamma) \wedge \operatorname{CARD}(\gamma)>3)$ 
c. exactly three students $\sim \mathrm{BE}$ (exactly three students)

$\lambda Q \exists \gamma\left(\operatorname{Student}(\gamma) \wedge \operatorname{CARD}(\gamma)=3 \wedge{ }^{\vee} Q(\gamma)\right) \sim_{B E}$

$\lambda \gamma(\operatorname{Student}(\gamma) \wedge \operatorname{CARD}(\gamma)=3)$

In these representations, $\gamma$ is a variable over plural individuals and CARD is a predicate which counts the number of atoms that make up the sum. Along these lines, an atomic individual can be considered as an individual of cardinality one. Depending on whether we want to give the bare numeral NP three students an interpretation in terms of 'at least three' or 'exactly three', we can treat this NP as in (13a) or (13c). Note that there is some debate in the literature as to whether we want to assign modified numerals a group-level denotation on a par with bare numerals or not. Given that this discusssion plays more of a role in the distinction between type I and type II indefinites, I will simply assume at this point that we can. Further motivation of this decision is found in section 4 below.

Under the analysis outlined in this section, both weak NPs and definite NPs have well-formed denotations in the domain of expressions of type $\langle e, t\rangle$ after application of the type-shifter BE. However, definite NPs are special in that their type $\langle e, t\rangle$ denotation is always a singleton set. It is the unique individual (atomic or non-atomic) which is collected into a set by BE which guarantees this. Thus we can define the set of weak NPs as those NPs which, after application of $\mathrm{BE}$ have a well-formed type $\langle e, t\rangle$ denotation which is not a singleton set in all models under all assignment functions:

- Class I indefinites are those NPs which, after application of BE have a well-formed (i.e. non-empty) type $\langle e, t\rangle$ denotation which is not a singleton set in all models under all assignment functions.

The set of weak NPs defined on the basis of this criterion includes increasing, decreasing and non-monotone expressions as desired.

Once we know what the semantic type is of a weak indefinite NP, we need to think about how to combine the predicative denotation of the NP with other expressions in the sentence. At this point, we have two options. If we assume that weak subjects/objects require a non-quantificational treatment and are not to be treated as generalized quantifiers, even at the compositional level, we should find other ways of composition than classical function application. Carlson (1978), Heim (1982), Van Geenhoven (1996) and others have formulated alternative modes of composition which build a VP from a regular transitive verb and a predicative NP, or a proposition from the combination of a predicative NP and a VP. It is possible to work out such closure operations in a flexible type theory (compare de Swart 1999), but I will not do that here. Instead, I will stick to classical function application as the only mode of composition and stay as close as possible to the type-shifting principles defined by Partee (1987). If we assume that verbs normally select for NPs of type $e$ or type $\langle\langle s,\langle e, t\rangle\rangle, t\rangle$, the restriction to function composition means that property 
denoting NPs need to be shifted back to a generalized quantifier type before we can combine them with the verb or verb phrase. This requires the definition of inverse operations of $\mathrm{BE}$.

\section{From properties to generalized quantifiers}

\subsection{Pairs of inverse type-shifters}

Type-shifting operations typically come in pairs. One such pair is the relation between 'lift' and 'lower'. The 'lift' operation was introduced by Montague to assign proper names of type $e$ a generalized quantifier denotation of type $\langle\langle s,\langle e, t\rangle\rangle, t\rangle(14 \mathrm{a})$ :
a. lift: $a \leadsto \lambda P^{\vee} P(a)$
b. lower $(\operatorname{lift}(a))=a$

The inverse of lift is the 'lower' operation which maps ultrafilters onto their generator $(14 \mathrm{~b})$. With lower we can get back from the generalized quantifier interpretation of proper names to their referential interpretation. The lower operation is restricted to ultrafilters, which are generated by individuals. Partee is particularly interested in such pairs of inverse relations, because they relate denotations of one and the same NP in different domains.

If we are looking for inverse relations of $\mathrm{BE}$, we can raise two questions:

- Inverse relations of $\mathrm{BE}$

a. For which determinator denotations DET is it the case that $\mathrm{BE}(\mathrm{DET}(\mathrm{P}))=\mathrm{P}$ ?

b. For which determinator denotation DET is it the case that $\operatorname{DET}(\mathrm{BE}(\mathrm{NP}))=\mathrm{NP}$ ?

The derivation in (12) shows that the general answer to the first question is $a$. For the special case in which the type $\langle e, t\rangle$ denotation involves a singleton set, the answer is the. Partee therefore concludes that $\mathrm{BE}$ and $a /$ the are inverse operations. In a footnote, she points out that this answers just one of the questions after inverse operations of $\mathrm{BE}$. The other question we can ask is which determiner denotation DET guarantees that $\operatorname{DET}(\mathrm{BE}(\mathrm{NP}))=\mathrm{NP}$. According to Partee, it is impossible to give a general answer to this question. The reason is that with the application of $\mathrm{BE}$ we lose information. In particular, $\mathrm{BE}\left(\mathrm{NP}_{1}\right)$ $=\mathrm{BE}\left(\mathrm{NP}_{2}\right)$ for every $\mathrm{NP}_{1}$ and $\mathrm{NP}_{2}$ which contain the same singleton sets in their denotation. For example, a student, exactly one student and less than two students contain the same singleton sets of one student in their denotation. Any attempt to recover the original generalized quantifier denotation on the basis of the union of these singleton sets is thus doomed to fail. 
Although Partee's observations are quite correct for the general case, I do not think that we should therefore abandon the search. It turns out to be quite possible to define inverse operations for subclasses of NPs if we do not just look at the set of individuals which result from the application of $\mathrm{BE}$, but if we take into consideration certain properties of the generalized quantifier denotation which BE operates on. More specifically, I propose to define partial functions, and make the inverse operation dependent on the monotonicity properties of the underlying NP.

\subsection{Insertion operations}

For monotone increasing NPs, the insertion of an existential quantifier (over individuals or groups) is the appropriate way to restore the original generalized quantifier denotation. I call this operation existential insertion $(\exists \mathrm{I})$, and make the following generalization:

(15) For mon $\uparrow \mathrm{NPs}, \exists \mathrm{I}(\mathrm{BE}(\mathrm{NP}))=\mathrm{NP}$, where $\exists \mathrm{I}$ is defined as follows: $\exists \mathrm{I}: \lambda P \lambda Q \exists \gamma\left({ }^{\vee} Q(\gamma) \wedge{ }^{\vee} P(\gamma)\right)$

The insertion operation defined in (15) is of type $\langle\langle s,\langle e, t\rangle\rangle,\langle\langle s,\langle e, t\rangle\rangle, t\rangle\rangle$, the type of a determiner denotation. The result of the application of $\exists \mathrm{I}$ to a property $P$ is a generalized quantifier which expresses existential quantification over the elements that are a member of the set of individuals or groups which $P$ denotes. The variable $Q$ stands for the VP-denotation. $\exists \mathrm{I}$ is thus nothing but the determiner $a$ suitably generalized to non-atomic individuals. A version of existential insertion as a type-shifting operation mapping set-denoting NPs onto generalized quantifiers is already defined by Bittner (1994), Landman (1998) and Chierchia (1998). It is used to preserve function application as the principal mode of composition. In a similar vein, Winter (1997) develops a type-shifted version of choice functions, which is of the same semantic type as the insertion operation defined here. Some examples of restauration of the original generalized quantifier denotation by $\exists I$ are given in (16), where the type-shifting operation is indicated by an arrow $\sim_{\exists I}$ :

(16) a. $\mathrm{BE}($ a genius) $\sim \exists I$ a genius

$\lambda x \operatorname{Genius}(x) \sim \exists I \lambda P \lambda Q \exists \gamma\left({ }^{\vee} Q(\gamma) \wedge{ }^{\vee} P(\gamma)\right)\left({ }^{\wedge} \lambda x \operatorname{Genius}(x)\right)$

$=\lambda Q \exists x\left({ }^{\vee} Q(x) \wedge \operatorname{Genius}(x)\right)$

b. $\mathrm{BE}$ (at least three students) $\sim \exists I$ at least three students

$\lambda \gamma(\operatorname{Student}(\gamma) \wedge \operatorname{CARD}(\gamma) \geq 3) \sim \exists I$

$\lambda Q \exists \gamma\left({ }^{\vee} Q(\gamma) \wedge \operatorname{Student}(\gamma) \wedge \operatorname{CARD}(\gamma) \geq 3\right)$

The insertion of an existential quantifier accounts for the intuition that the predicative interpretation of a mon $\uparrow$ NP describes a 'minimal' property. For example, at least three students implies that four or more is also allowed. 
To my knowledge, no one has defined inverse operations of $\mathrm{BE}$ for nonincreasing indefinites. Obviously, $\exists \mathrm{I}$ cannot be used to recover the original generalized quantifier denotation of mon $\downarrow$ NPs, because the predicative interpretation of these NPs describes a 'maximal' property. For example, at most five students implies that four or less is also admissible. The notion of maximality naturally suggests an operation of universal insertion $(\forall \mathrm{I})$ as the inverse of BE for mon $\downarrow$ NPs:

(17) For mon $\downarrow$ NPs, $\forall \mathrm{I}(\mathrm{BE}(\mathrm{NP}))=\mathrm{NP}$, where $\forall \mathrm{I}$ is defined as follows:

$\forall \mathrm{I}: \lambda P \lambda Q \forall \gamma\left({ }^{\vee} Q(\gamma) \rightarrow{ }^{\vee} P(\gamma)\right)$

Just like the insertion operation defined in (15), $\forall \mathrm{I}$ is of type $\langle\langle s,\langle e, t\rangle\rangle,\langle\langle s,\langle e, t\rangle\rangle, t\rangle\rangle$, i.e. the type of a determiner denotation. Some examples of the application of $\forall \mathrm{I}$ are given in (18):

a. $\mathrm{BE}$ (no genius) $\sim_{\forall I}$ no genius

$\lambda x \neg \operatorname{Genius}(x) \sim_{\forall I}$

$\lambda P \lambda Q \forall \gamma\left({ }^{\vee} Q(\gamma) \rightarrow{ }^{\vee} P(\gamma)\right)(\wedge \lambda x \neg \operatorname{Genius}(x))$

$=\lambda Q \forall x\left({ }^{\vee} Q(x) \rightarrow \neg \operatorname{Genius}(x)\right)$

b. $\mathrm{BE}$ (at most five students) $\sim_{\forall I}$ at most five students

$\lambda \gamma \neg(\operatorname{Student}(\gamma) \wedge \operatorname{CARD}(\gamma)>5) \sim \forall I$

$\lambda Q \forall \gamma\left({ }^{\vee} Q(\gamma) \rightarrow \neg(\operatorname{Student}(\gamma) \wedge \operatorname{CARD}(\gamma)>5)\right)$

Finally, non-monotone NPs such as exactly one student involve a mixture of minimality and maximality conditions. This is appropriately captured by a type-shifting operation which inserts an exact existential quantifier. The definition is given in (19); an application to an example is found in (20):

(19) For non-monotone NPs, built on a common noun $\mathrm{CN}, \exists ! \mathrm{I}(\mathrm{BE}(\mathrm{NP}))=$ NP, where $\exists ! I$ is defined as follows:

$\exists ! I: \lambda P \lambda Q \exists \gamma\left({ }^{\vee} Q(\gamma) \wedge{ }^{\vee} P(\gamma) \wedge \forall \gamma^{\prime}\left(\left({ }^{\vee} Q\left(\gamma^{\prime}\right) \wedge{ }^{\vee} C N\left(\gamma^{\prime}\right)\right) \rightarrow \gamma^{\prime} \sqsubseteq \gamma\right)\right)$

(20) a. BE(exactly one student) $\sim_{\exists ! I}$ exactly one student

$\lambda \gamma(\operatorname{Student}(\gamma) \wedge \operatorname{CARD}(\gamma)=1) \sim_{\exists ! I}$

$\lambda P \lambda Q \exists \gamma\left({ }^{\vee} Q(\gamma) \wedge{ }^{\vee} P(\gamma) \wedge \forall \gamma^{\prime}\left(\left({ }^{\vee} Q\left(\gamma^{\prime}\right) \wedge{ }^{\vee} C N\left(\gamma^{\prime}\right)\right) \rightarrow\right.\right.$

$\left.\left.\gamma^{\prime} \sqsubseteq \gamma\right)\right)(\wedge \lambda \gamma \operatorname{Student}(\gamma) \wedge \operatorname{CARD}(\gamma)=1)$

$=\lambda Q \exists \gamma\left({ }^{\vee} Q(\gamma) \wedge \operatorname{Student}(\gamma) \wedge \operatorname{CARD}(\gamma)=1 \wedge\right.$

$\left.\forall \gamma^{\prime}\left(\left({ }^{\vee} Q\left(\gamma^{\prime}\right) \wedge \operatorname{Student}\left(\gamma^{\prime}\right)\right) \rightarrow \gamma^{\prime} \sqsubseteq \gamma\right)\right)$

b. BE(exactly three students) $\sim_{\exists ! I}$ exactly three students

$\lambda \gamma(\operatorname{Student}(\gamma) \wedge \operatorname{CARD}(\gamma)=3) \sim \exists ! I$

$\lambda P \lambda Q \exists \gamma\left({ }^{\vee} Q(\gamma) \wedge{ }^{\vee} P(\gamma) \wedge \forall \gamma^{\prime}\left(\left({ }^{\vee} Q\left(\gamma^{\prime}\right) \wedge{ }^{\vee} C N\left(\gamma^{\prime}\right)\right) \rightarrow\right.\right.$

$\left.\left.\gamma^{\prime} \sqsubseteq \gamma\right)\right)\left({ }^{\wedge} \lambda \gamma \operatorname{Student}(\gamma) \wedge \operatorname{CARD}(\gamma)=3\right)$

$=\lambda Q \exists \gamma\left({ }^{\vee} Q(\gamma) \wedge \operatorname{Student}(\gamma) \wedge \operatorname{CARD}(\gamma)=3 \wedge\right.$

$\left.\forall \gamma^{\prime}\left(\left({ }^{\vee} Q\left(\gamma^{\prime}\right) \wedge \operatorname{Student}\left(\gamma^{\prime}\right)\right) \rightarrow \gamma^{\prime} \sqsubseteq \gamma\right)\right)$ 
The examples show that there is a consistent relation between mon $\uparrow$ NPs and 'minimality' on the one hand, and mon $\downarrow$ NPs and 'maximality' on the other hand. Non-monotone NPs combine the two and require the group of individuals who satisfies both the $\mathrm{CN}$ and the VP-denotation to consist of exactly the number of individuals given by the numeral. The operations of existential, universal and exact existential insertion capture this correlation, and make it possible to develop a systematic treatment of converse relations of $\mathrm{BE}$ in a type-shifting framework. In sum, we can answer the question which $\mathrm{DET}$ is such that $\operatorname{DET}(\mathrm{BE}(\mathrm{NP}))=\mathrm{NP}$ as follows:

- For monotone increasing NPs, $\exists \mathrm{I}(\mathrm{BE}(\mathrm{NP}))=\mathrm{NP}$

- For monotone decreasing NPs, $\forall \mathrm{I}(\mathrm{BE}(\mathrm{NP}))=\mathrm{NP}$

- For non-monotone NPs, $\exists ! \mathrm{I}(\mathrm{BE}(\mathrm{NP}))=\mathrm{NP}$

The fact that we have to make $\exists I$ and $\forall \mathrm{I}$ sensitive to the monotonicity properties of the underlying NP makes these type-shifting operations much more specific than the operation $\mathrm{BE}$ they are the converse of. This raises the question whether such partial functions are 'natural' enough to be available in a general type-shifting framework. At least one other example of a partial typeshifting operation is discussed by Partee (1987), namely the lower operation. It is well known that the lift operation in (15a) can be generalized to expressions of any type. However, its inverse relation, the lower operation in (15b) is only defined for ultrafilters, i.e. generalized quantifiers that have an individual as their generator. This is another instance in which properties of the argument of the functor are relevant for the definition of the inverse of that functor. The formulation of restrictions on $\exists \mathrm{I}$ and $\forall \mathrm{I}$ in terms of the monotonicity properties of the NP on which BE operates are similar in nature. Notwithstanding their more specific nature, there is thus sufficient reason to consider the partial operations $\exists \mathrm{I}, \forall \mathrm{I}$ and $\exists$ !I as instances of 'natural' type-shifting operations.

\section{Referentiality}

\subsection{Increasing monotonicity as a necessary condition}

If $\exists$ I, $\forall \mathrm{I}$ and $\exists$ !I are freely available type-shifting operations which come into play at the compositional level, we do not yet have an explanation for the fact that (some) predicative NPs which undergo $\exists I$ license discourse anaphora, whereas predicative NPs which undergo $\forall \mathrm{I}$ and $\exists$ !I do not. The insertion operations themselves do not account for the different dynamic binding properties of these expressions, illustrated in (7) above, and repeated here as (21):

(21) a. A student ${ }_{i}$ called. She ${ }_{i}$ had a question about the exam

b. Every student ${ }_{i}$ called. \# $\mathrm{She}_{i}$ had a question about the exam 
c. No student ${ }_{i}$ called. \# $\mathrm{She}_{i}$ had a question about the exam

A straightforward and attractive solution to the problem of restricting class II indefinites to mon $\uparrow$ indefinites is to adopt the proposals made by Groenendijk and Stokhof (1990, 1991). Groenendijk and Stokhof develop dynamic versions of predicate logic and Montague grammar in which all the NPs in (21) denote generalized quantifiers. However, different generalized quantifiers have different dynamic properties. In particular, the existential quantifier gets an externally dynamic interpretation. As a result, an anaphoric pronoun in a later sentence can refer to the same individual. Unlike the existential quantifier, the universal quantifier is normally externally static, and its scope does not extend beyond the sentence in which it occurs.

The insertion operations defined in section 3 combine well with the dynamic approach, because the inverse relations of $\mathrm{BE}$ return a generalized quantifier denotation. This way, we can build the right binding properties into a dynamic version of the type-shifting approach. Both $\forall \mathrm{I}$ and $\exists$ !I introduce universal quantification over the variable introduced by the NP. This will lead to an externally static generalized quantifier. $\exists \mathrm{I}$ is the only insertion operation which relies exclusively on existential quantification over the variable introduced by the NP. Thus, if we assume that existential quantification is externally dynamic, but universal quantification is not, we can argue that increasing monotonicity is a necessary condition for referentiality.

\subsection{Referentiality as mapping onto a type e denotation}

Although it is true that no monotone decreasing and non-monotone quantifiers license discourse anaphora, it is not true that all monotone increasing quantifiers do. Crucially, the set of plural NPs is more heterogeneous. The examples in (8), repeated here as (22) illustrate that some $\mathrm{N}$, many $\mathrm{N}$ and bare numeral NPs license non-maximal discourse anaphora, NPs like at least five $\mathrm{N}$ or more than five $\mathrm{N}$ only allow maximal anaphora, as argued by Kamp and Reyle (1993), Szabolcsi (1997a, b) and others:

(22) a. Two students came in. They had a question about the exam.

b. At least two students came in. They had a question about the exam.

c. Exactly two students came in. They had a question about the exam.

d. All students came to the meeting. They requested a new class schedule.

The criterion most of ten used to establish the distinction between the two classes is the possibility of getting a collective reading (Kamp and Reyle 1993, Winter 1998, Honcoop 1998). Bare numeral NPs easily allow collective or cumulative readings (23a), whereas for modified numeral NPs, the distributive reading is usually preferred $(23 \mathrm{~b})$ : 
(23) a. Two students drank a glass of beer together.

b. \# At least ten students drank a glass of beer together.

For Kamp and Reyle (1993), the distinction between (23a) and (23b) motivates a rather different treatment of bare numeral NPs and modified numeral NPs. Bare numerals like two students are treated like indefinites. Modified numerals like at least ten students are treated like cardinal quantifiers, with properties similar to generalized quantifiers. Honcoop (1998) and Winter (1998) establish a similar distinction in dynamic Montague grammar and the choice function approach respectively. A related proposal is made by Szabolcsi (1997a: 25), who takes bare numeral NPs, but not modified numeral NPs to have a referential reading. She characterizes referential indefinites as principal filters, on a par with proper names and definite descriptions.

The intuition underlying these proposals is that the NP somehow needs to be able to pick out a singular or plural individual in order to count as a class II indefinite. Once we have an individual we can predicate over, we can interpret predicates later in the discourse as further predications over this same individual. Thus referential indefinites license discourse anaphora. In some intuitive sense, their referential nature also makes these indefinites scopeless. This underlies their capacity to escape out of scope islands and to be harmless interveners in weak island constructions. In type-theoretical terms, the intuition that referential NPs refer to individuals corresponds with the claim that they have a well-formed denotation of type $e$ (cf. Partee 1987). The question which remains to be answered is which NPs have a type $e$ denotation, and why. In particular, we would like to know why bare numerals have a type $e$ denotation, but modified numerals do not. We cannot claim that modified numerals only have a generalized quantifier denotation, because we know from section 2 above that they also have a felicitous type $\langle e, t\rangle$ denotation. Denying modified numerals a non-quantificational interpretation would make it impossible to account for their weak, predicative interpretation, so that road is closed to us. If we assume that referential NPs have a type $e$ denotation, the real question involves determining the constraints on mappings from type $\langle e, t\rangle$ onto $e$, and from $\langle\langle e, t\rangle, t\rangle$ onto $e$. It is these constraints which should explain the difference between bare and modified numerals. Unlike the domain of type $\langle e, t\rangle$ or type $\langle\langle e, t\rangle, t\rangle$ expressions, the domain of expressions of type $e$ does not have Boolean structure. This implies that the constraints on the mapping onto type $e$ are probably of a different nature from what we have seen so far. This leads me to propose an ontological explanation in terms of the structure of the domain of (plural) individuals.

In section 2 above, we argued that we need to assume Link's (1983) latticetheoretical ontology of plurals. The join semi-lattice of a set of four individuals is pictured in figure 2: 
figure 2

Join semi-lattice of a set of four individuals in the denotation of some plural common noun $\mathrm{N}^{*}$ (stylized version):

a.

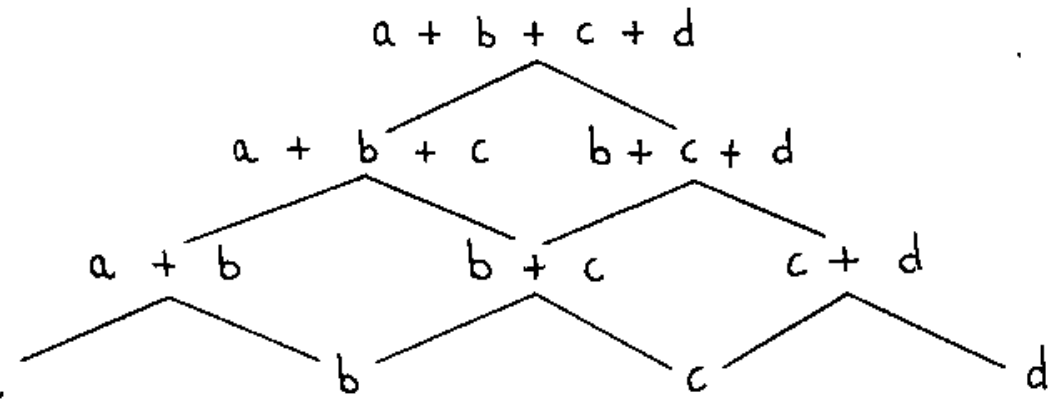

The top, the maximum of the lattice is a plural individual which is the denotation of the $\mathrm{N}^{*}$ or the four $\mathrm{N}^{*}$. Everything below the top has more than one individual on each row. This reflects a basic distinction between definite and indefinite NPs: definite NPs pick out a (contextually) unique referent, indefinite NPs potentially leave us a choice of referent. The bottom row gives us the set of atomic individuals in the extension of $\mathrm{N}^{*}$ (this set is the extension of $\mathrm{N}$ ). The row above that gives us all the plural individuals which consist of a sum of two individuals, the row above that contains sums of three individuals, etc. Each row thus consists of individuals of the exact same size. The size seems to provide an identity criterion for the individual: each row defines individuals of cardinality $n$. In this perspective, we can take singular NPs of the form $a \mathrm{~N}$ to pick out an atomic individual from the bottom row. Bare numeral NPs pick out non-atomic individuals from one of the rows above the bottom one. What we should emphasize is that two $\mathrm{N}^{*}$, three $\mathrm{N}^{*}$, etc. all pick out their own row in the lattice:

figure 3

Bare numerals: $\mathrm{n} \mathrm{N}^{*}$

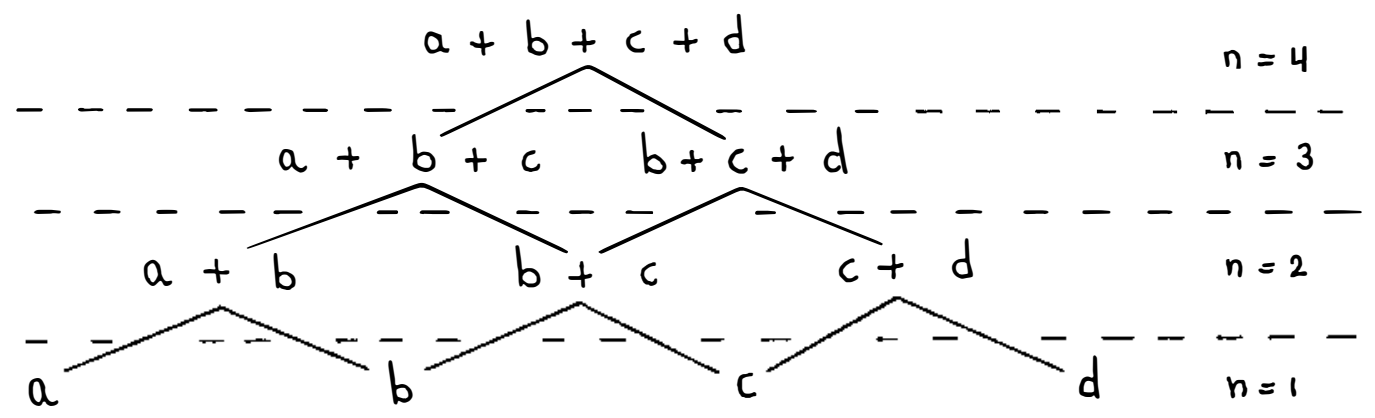

Now compare this picture with the one generated by modified numeral NPs like at least three $\mathrm{N}^{*}$, more than five $\mathrm{N}^{*}$. These NPs do not pick out rows, but sublattices: they pick out everything from a particular row upwards: 
figure 4

modified numerals: at least three $\mathrm{N}^{*}$

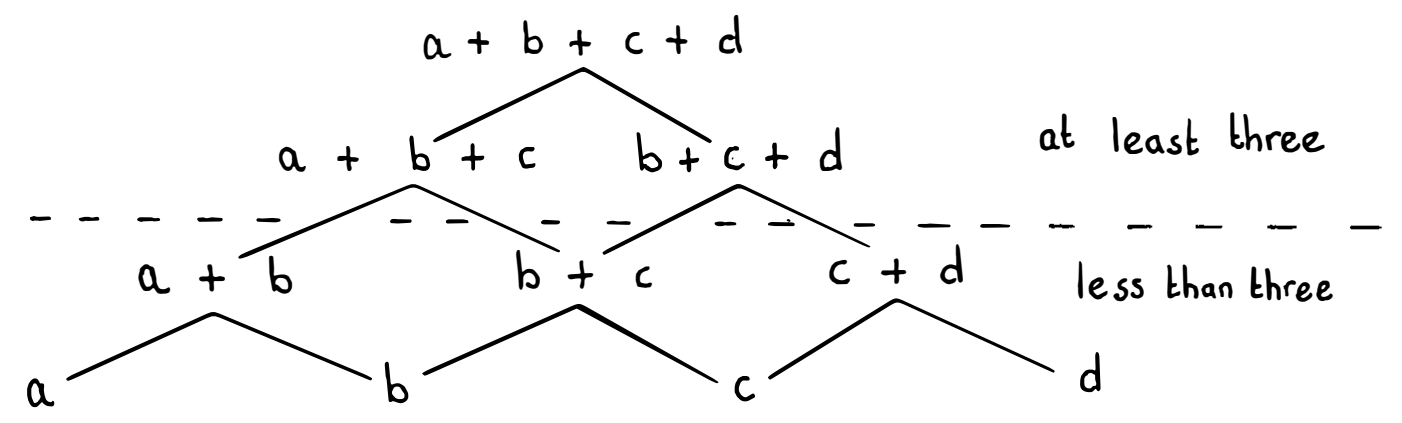

The intuition is that at least $n$ does not provide a good identity criterion, for the members of the set of individuals which make up the denotation of the NP are much too heterogeneous in nature. If we cannot use the NP to pick out and identify an individual, it does not have a well-formed type $e$ denotation. We conclude that modified numerals do not have a well-formed type $e$ denotation under the 'row' identity perspective.

The formal counterpart of the 'row' identity criterion is a partitioning of the domain of individuals in equivalence classes of individuals consisting of the same number of atoms. A partition B of a set B is a set of non-empty subsets of B such that the union of those subsets equals B and no two of these subsets overlap (Groenendijk and Stokhof 1984: 214, Partee et al. 1990: 46):

(24) $\mathrm{B}$ is a partition of a non-empty set $\mathrm{B}$ iff $\forall X \in B$ it is the case that:

1) $X \neq \emptyset$ and $\cup X \in \mathrm{B}=B$ and

2) $\forall X \forall Y \in \mathrm{B}: X \cap Y=\emptyset \vee X=Y$

The subsets that are members of a partition are called cells of that partition. By dividing a set into mutually exclusive and collectively exhaustive nonempty subsets, we can effect any partitioning of the set that we want. The most interesting partitions are those based on some 'natural' identity criterion for the cells. The identity criterion takes the form of an equivalence relation. To every equivalence relation $R$ on a set $B$ corresponds a partition of $B$, the elements being the equivalence classes of $B$ under $R$ (Groenendijk and Stokhof 1984: 215-216; Partee et al. 1990: 46-47). For the case at hand, we create a partitioning of the domain of individuals based on the 'size' of the individual, that is, the number of atomic individuals that constitutes the (possibly plural) individual. Therefore, we use the relation 'be made up of the same number of atomic individuals' to partition the domain of individuals D. Call this relation $\mathrm{S}$ (for 'same size'). The relation $\mathrm{S}$ is reflexive, symmetric and transitive, i.e. it is an equivalence relation. Using the relation $S$ thus partitions the domain of individuals $\mathrm{D}$ into sets of individuals which have the same number of atoms 
the individual is made up of. Consequently, within each cell, individuals are of the exact same size. Based on the equivalence relation $S$, we obtain the following partition $\mathrm{D}$ of $\mathrm{D}$ :

figure 5

Partition D induced by S ('same size')

\begin{tabular}{|l|}
\hline$\ldots$ \\
\hline sums of four individuals \\
\hline sums of three individuals \\
\hline sums of two individuals \\
\hline atomic individuals \\
\hline
\end{tabular}

The partition $\mathbf{D}$ distinguishes between individuals of different sizes. Singular individuals are atomic individuals. Plural individuals are non-atomic individuals of a particular size. We can now characterize referential NPs by saying that they identify a particular cell in the partition D. Definite NPs pick out the unique element in the top cell of $\mathbf{D}$. This is encoded by the mapping iota (cf. figure 1). For indefinites, the cell may contain more individuals, but the choice is made among individuals of the same size. Whether we do this via a choice function, a DRT embedding function or a DMG assignment function is irrelevant at this point. In figure 6 below, the arrow is labelled c(hoice) $\mathrm{f}$ (unction) for convencience. What is more important is that indefinites which pick out an arbitrary element from a particular cell in the partition $\mathbf{D}$ satisfy the identity criterion given by the equivalence relation S. NPs which satisfy the identity criterion are NPs which have a well-formed type $e$ denotation. This allows us to characterize $a \mathrm{~N}$ and bare numeral NPs as class II indefinites. Modified numerals do not satisfy the identity criterion under the partition $\mathbf{D}$, induced by $\mathrm{S}$. Thus they do not have a well-formed type $e$ denotation in this perspective. If we take $\mathrm{S}$ to be the default setting, we can explain the difference between bare and modified NPs in ontological terms. We can treat some $\mathrm{N}$ and many $\mathrm{N}$ along similar lines if we allow some room for vagueness in the way the partition is built up.

We can establish a connection with dynamic logic by linking the externally dynamic behavior of indefinites to their ontology. NPs which have a well-formed denotation of type $e$ are externally dynamic, NPs which don't are externally static. This leads to an externally dynamic definition of the bare numeral two students in (25a), and an externally static definition of the modified numeral at least two students in $(25 \mathrm{~b})$ :

(25) a. two students $=$

$$
\begin{aligned}
& \mathcal{E} d(\uparrow \operatorname{Student}(d) ; \uparrow \operatorname{Card}(d)=2)= \\
& \lambda p \exists \gamma\left(\operatorname{Student}(\gamma) \wedge \operatorname{Card}(\gamma)=2 \wedge[\gamma / d]^{\vee} p\right)
\end{aligned}
$$


b. at least two students $=$ $\uparrow \downarrow \mathcal{E} d(\uparrow \operatorname{Student}(d) ; \uparrow \operatorname{Card}(d) \geq 2)=$ $\lambda p \exists \gamma\left(\operatorname{Student}(\gamma) \wedge \operatorname{Card}(\gamma) \geq 2 \wedge{ }^{\vee} p\right)$

The externally dynamic character of the existential quantifier in (25a) leads to the anchoring of all occurrences of the discourse referent $d$ in subsequent sentences to the same individual $x$, which is a plural student individual. The application of $\uparrow \downarrow$ to this dynamic formula in (25b) leads to existential quantification in the truth conditions, but removes all dynamic effects. Thus, the discourse referent $d$ does not 'live on' beyond the sentence boundary. The introduction of externally static existential quantifiers next to externally dynamic existential quantifiers allows us to account for the fact that all monotone increasing quantifiers involve existential quantification as part of their truth conditions, but not all monotone increasing quantifiers are externally dynamic. The claim made in this section is thus that the externally dynamic behavior of an expression is not exclusively determined by truth conditions, but is ultimately dependent on the question whether we can project the NP onto a type $e$ denotation.

\section{More dynamics than we thought}

The introduction of externally static existential quantifiers next to externally dynamic ones opens up new perspectives for the study of modified numerals. It has been observed that quantifiers and connectives which are normally externally static can display dynamic behavior in certain contexts. For universal quantifiers and negation this is illustrated in (26) and (27):

(26) Every player $_{i}$ chooses a pawn p $_{j} \mathrm{He}_{i}$ puts it ${ }_{j}$ on square one.

(27) a. It is not true that John doesn't own a car . $_{i} \mathrm{It}_{i}$ is red, and it is parked outside.

b. Either there is no bathroom $i$ in this house, or it ${ }_{i}$ is in a weird place.

For analyses, compare Groenendijk and Stokhof (1989), Dekker (1993), Krahmer and Muskens (1995) and others. The technical details of these proposals do not concern us here, but the data raise interesting new questions for our treatment of modified numeral NPs as externally static quantifiers. If we can find dynamic behavior for normally externally static operators such as universal quantifiers and negation, could we also find dynamic behavior for modified numerals, which we treated as essentially externally static? The standard assumption is that cardinal quantifiers do not license non-maximal discourse anaphora (Kamp and Reyle 1993, Szabolcsi 1997a,b). However, Szabolcsi (1997b) discusses Hungarian examples which seem to allow for a nonmaximal interpretation of modified numerals (at least when they occur in certain syntactic positions): 
(28) Több, mint hat diákunk félreértette a kérdést. Lehet, hogy még másokat is találsz.

More than six of our students misunderstood the question. Maybe you will find others too.

The fact that the continuation is felicitous suggests that non-maximal reference is possible. However, the distinction between maximal and non-maximal discourse anaphora is subtle, and it can be hard to draw the line. Sentenceinternal anaphora might provide a better testcase. If cardinal NPs are externally static, we do not expect them to license (non-maximal) donkey anaphora. However, if we compare the sentences in (29), it seems that the modified numerals more than twenty students (29c) and exactly 100 points (29d) behave like their bare counterpart twenty students (29a), rather than the quantificational plural NP most students (29b):

(29) a. If you have twenty students , you can split them $_{i}$ into two groups.

b. *If most students ${ }_{i}$ signed up for your class, you can split them ${ }_{i}$ into two groups.

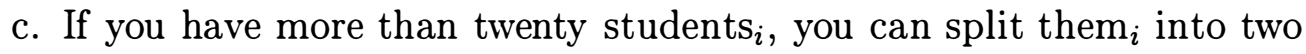
groups.

d. If you end up with exactly 100 points $_{i}$ you can use them $i$ to get an extra turn in the next round.

The anaphora in (29a), (c) and (d) have a non-maximal interpretation: one can have exactly 100 points more than once during a game, and if 40 or 50 students take the class, even more groups than two will be created.

Similarly, the monotone decreasing modified numeral less than five students in $(30 \mathrm{c})$ behaves like the negative plural no students in (30a), rather than the negative plural quantifier not all students in (30b):

(30) a. Either there are no students $i$ who registered for this class, or they $i$ went to the wrong room.

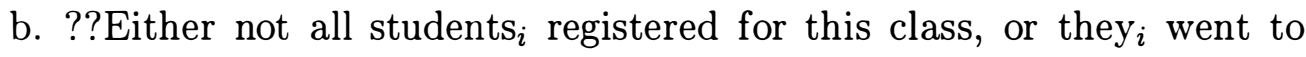
the wrong room.

c. Either there are less than five students $i$ who registered for this class, or they ${ }_{i}$ went to the wrong room.

d. It is not the case that only few students $i$ registered for this class. In fact, they $y_{i}$ are so numerous, they ${ }_{i}$ don't fit into the room. 
The pronoun they in (30a) and (c) picks out the set of students who registered for this class. Moreover, for (30c) this set contains more than five individuals, and for (30d), this set contains more than a small numer of individuals. This kind of anchoring process is impossible in (30b), because the antecedent is a real quantificational NP.

The data in (29) and (30) suggest that modified numerals are not as externally static as real quantificational NPs. The question remains why they are not as externally dynamic as bare numerals. Once more, the answer resides in the ontology. We argued that bare numerals like two students are externally dynamic, because they can get a type $e$ denotation. They can get a type $e$ denotation, because we can partition the domain in such a way that the NP picks out an arbitrary individual from a particular cell of the partition $\mathbf{D}$. The partition $\mathbf{D}$ is induced by the equivalence relation $\mathrm{S}$ 'to be the same size as', and $S$ is a natural relation to build a partition on, because the lattice-theoretical ontology of plurals is based on the join operation. Even if that makes $\mathrm{S}$ the default choice, it is clear that $\mathrm{S}$ is not the only relation we could build a partition on. Note that the example in (29d) is felicitous in the context of a game in which no number besides 100 will lead to the desired extra turn. This means that the relevant partition is a partition $\mathbf{D}^{\prime}$, which is based on the equivalence relation $S$ ' ('share the property of having exactly $n$ members' ${ }^{6}$. The partition $\mathbf{D}^{\prime}$ consists of two cells: sums of $n$ individuals and sums of more or less than $n$ individuals. The other examples in (29) and (30) can be accounted for along similar lines. They all involve a context in which a particular number functions as the cut-off point: all groups with less members fall in one category and groups with more members fall into the other one. Obviously, such a cut-off point can be used to partition the domain of individuals into two subsets which are mutually exclusive: the set of individuals with cardinality greater than (or equal to) some number $n$, and the set of individuals with cardinality smaller than (or equal to) $n$. This partition D" is based on the equivalence relation S", which can be defined as 'sharing the property of having $n$ or more atomic members'. S" induces the partition D" of our domain of individuals into two cells of $n$ or more individuals and less than $n$ individuals. Note that the sublattice of figure 4 has now been projected onto a cell of a partition. If the context favors a partition like $\mathbf{D}^{\prime}$ or D", rather than the default partition $\mathbf{D}$, the modified numeral can pick out an arbitrary member of a particular cell of the partition. As a result, the NP satisfies the identity criterion, and we can assign it a type $e$ denotation. The type $e$ denotation induces the externally dynamic behavior which accounts for the anaphoric licensing in (29) and (30). The conclusion must be that the external dynamic or static behavior of a plural indefinite NP depends on the partition that is salient in the context at hand. It is intuitively easy to see that S provides a more 'natural' partition than S' or S": the partitions D' and D" are degenerate in the sense that they involve only two cells. Only $\mathrm{S}$ provides a nice fine-grained partition into a larger number of cells. 


\section{Conclusion}

In this paper, I have tried to make sense of the different classifications of indefinite NPs we find in the literature by adopting Partee's (1987) flexible type theory as a starting point of the investigation. The type-shifting operations used in this analysis are summarized in figure 6:

figure 6 Type-shifting operations in the nominal domain

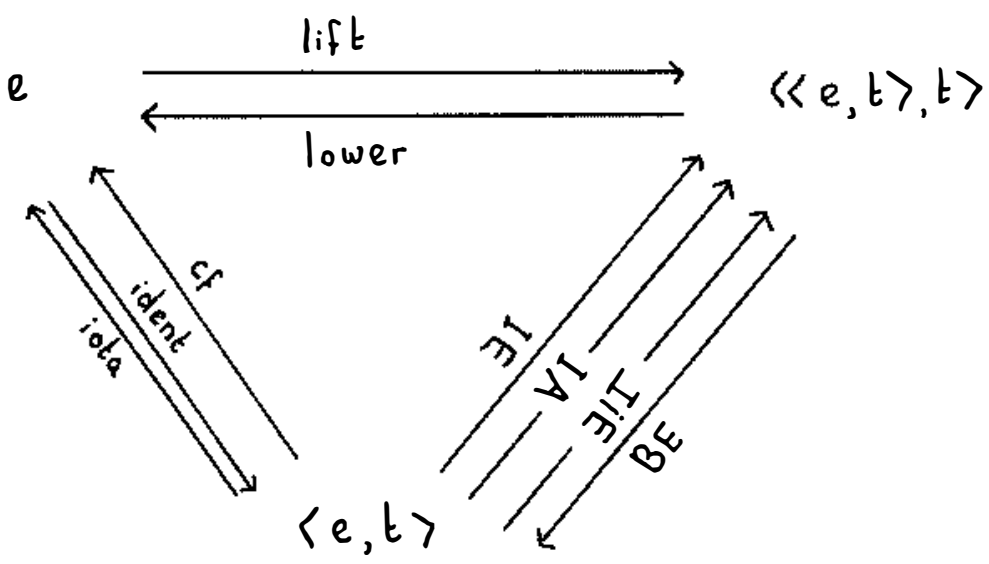

Class I indefinites have a non-empty predicative type $\langle e, t\rangle$ interpretation which does not correspond with a singleton set. The predicative type is derived by means of Partee's type shifter BE. The partial functions $\exists \mathrm{I}, \forall \mathrm{I}$ and $\exists$ !I all map predicative expressions back onto generalized quantifiers. The characterization of class II indefinites crucially involves referentiality. We defined referential indefinites as those NPs which allow projection of the generalized quantifier or predicative interpretation onto a type $e$ denotation. The externally dynamic properties of a plural NP are dependent on the partition of the domain of individuals that is salient in the context at hand. As a result, the distinction between bare and modified numerals becomes gradual, rather than absolute.

One of the advantages of the flexible type-theoretical framework is that it allows an integrated account of indefinites, which incorporates insights from different theoretical perspectives. Thus the ideas developed in this paper contribute to the discussion about the relation between theories like File Change Semantics, Discourse Representation theory, the choice function approach, and Dynamic Montague grammar.

\section{Endnotes}

* I thank the Linguistic Research Center of the University of California at Santa Cruz, and in particular Donka Farkas and Bill Ladusaw for their kind invitation to present this paper at SALT 9, and for their hospitality during 
my stay. Thanks also to the audience at SALT 9 for helpful comments on the first version of this paper. I am grateful to the Royal Dutch Academy of Sciences (KNAW) and the Dutch Linguistic Research School (LOT) for financially supporting this trip.

\section{References}

Abusch, D.: 1994, The scope of indefinites, Natural language semantics 3, $88-135$

Barwise, J. and Cooper, J.: 1981, Generalized quantifiers and natural language, Linguistics and Philosophy 4, 159-219

Beghelli, F. and Stowell, T.: 1997, Distributivity and negation: the syntax of each and every, in A. Szabolcsi (ed.), Ways of scope taking, pp 71-107, Kluwer Academic Publishers, Dordrecht

Bittner, M.: 1994, Cross-linguistic semantics, Linguistics and Philosophy 17, $53-108$

Blutner, R.: 1993, Dynamic generalized quantifiers and existential sentences in natural languages, Journal of Semantics 10, 33-64

Carlson, G.: 1978, Reference to kinds in English, Garland, New York

Chierchia, G.: 1998, Reference to kinds across languages, Natural Language Semantics 6, 339-405

Dekker, P.: 1993, Transsentential meditations, Ph.D. thesis, Un. of Amsterdam

Farkas, D.: 1981, Quantifier scope and syntactic islands, in $C L S$, Vol. 17, pp $59-66$

Farkas, D.: 1997, Evaluation indices and scope, in A. Szabolcsi (ed.), Ways of scope taking, pp 183-215, Kluwer Academic Publishers, Dordrecht

Groenendijk, J. and Stokhof, M.: 1984, Studies on the semantics of questions and the pragmatics of answers, Ph.D. thesis, University of Amsterdam

Groenendijk, J. and Stokhof, M.: 1990, Dynamic Montague grammar, in L. Kálman and L. Pólos (eds.), Papers from the second symposium on logic and language, pp 3-48, Akademiai Kaido, Budapest

Groenendijk, J. and Stokhof, M.: 1991, Dynamic predicate logic, Linguistics and Philosophy 14, 39-100

Heim, I.: 1982, The semantics of definite and indefinite NPs, Ph.D. thesis, University of Massachusetts, Amherst

Honcoop, M.: 1998, Dynamic excursions on weak islands, Ph.D. thesis, University of Leiden

de Hoop, H.: 1992, Case configuration and noun phrase interpretation, Ph.D. thesis, University of Groningen, published 1996 by Garland Press

de Jong, F.: 1987, The compositional nature of (in)definiteness, in E. Reuland and A. ter Meulen (eds.), The representation of (in)definiteness, pp 270285, MIT Press, Cambridge, MA

Kamp, H. and Reyle, U.: 1993, From discourse to logic, Kluwer, Dordrecht 
Krahmer, E. and Muskens, R.: 1995, Negation and disjunction in discourse representation theory, Journal of semantics 12, 357-367

Kratzer, A.: 1998, Scope or pseudoscope? are there wide-scope indefinites?, in S. Rothstein (ed.), Events and grammar, pp 163-196, Kluwer, Dordrecht

Landman, F.: 1998, Plurals and maximalization, in S. Rothstein (ed.), Events and grammar, pp 237-271, Kluwer Academic Publishers, Dordrecht

Link, G.: 1983, The logical analysis of plurals and mass terms, a latticetheoretic approach, in R. Bäuerle, C. Schwarze, and A. von Stechow (eds.), Meaning, use and interpretation of language, pp 302-323, de Gruyter, Berlin

McNally, L.: 1998, Existential sentences without existential quantification, Linguistics and Philosophy 21, 353-392

Milsark, G.: 1977, Toward an explanation of certain peculiarities of the existential construction of English, Linguistic Analysis 3, 1-29

Partee, B.: 1987, Noun phrase interpretation and type-shifting principles, in J. Groenendijk, D. de Jongh, and M. Stokhof (eds.), Studies in Discourse Representation theory and the theory of generalized quantifiers, pp 302-323, Foris, Dordrecht

Partee, B. H., ter Meulen, A., and Wall, R.: 1990, Mathematical methods in linguistics, Kluwer Academic Publishers, Dordrecht

Reinhart, T.: 1997, Quantifier scope: how labor is divided between QR and choice functions, Linguistics and Philosophy 20, 335-397

Ruys, E.: 1992, The scope of indefinites, Ph.D. thesis, Utrecht University

de Swart, H.: 1999, Weak readings of indefinites: type-shifting and beyond, ms. Utrecht University

de Swart, H.: to appear, Scope ambiguities with negative quantifiers, in U. Egli and K. von Heusinger (eds.), Reference and anaphorical relations, Kluwer Academic Publishers, Dordrecht

Szabolcsi, A.: 1997a, Background notions in lattice theory and generalized quantifiers, in A. Szabolcsi (ed.), Ways of scope taking, pp 1-27, Kluwer Academic Publishers, Dordrecht

Szabolcsi, A.: 1997b, Strategies for scope taking, in A. Szabolcsi (ed.), Ways of scope taking, pp 109-154, Kluwer Academic Publishers, Dordrecht

Van Geenhoven, V.: 1996, Semantic incorporation and indefinite descriptions, Ph.D. thesis, University of Tübingen

Winter, Y.: 1997, Choice functions and the scopal semantics of indefinites, Linguistics and Philosophy 20, 399-467

Winter, Y.: 1998, Flexible Boolean semantics, Ph.D. thesis, Utrecht University Zimmermann, E.: 1993, On the proper treatment of opacity in certain verbs, Natural Language Semantics 1, 149-179 